\title{
Manned Spacecraft Requirements for Materials and Processes
}

\author{
Timothy P. Vaughn.* \\ NASAMMarshall Space Flight Center, MSFC, AL 35812; Mail Code: EM30
}

\begin{abstract}
A major cause of project failure can be attributed to an emphasized focus on end products and inadequate attention to resolving development risks during the initial phases of a project. The initial phases of a project, which we will call the "study period", are critical to determining project scope and costs, and can make or break most projects. If the requirements are not defined adequately, how can the scope be adequately determined, also how can the costs of the entire project be effectively estimated, and how can the risk of project success be accurately assessed?
\end{abstract}

Using the proper material specifications and standards and incorporating these specifications and standards in the design process should be considered inherently crucial to the technical success of a project as just as importantly, crucial to the cost and schedule success. This paper will intertwine several important aspects or considerations for project success: 1) Characteristics of a "Good Material Requirement" 2) Linking material requirements to the implementation of "Design for Manufacturing" techniques and 3) The importance of decomposing materials requirements during the study phase/development phase to mitigate project risk for the maturation of technologies before the building of hardware.

\section{Introduction}

A erospace materials and processes requirements can be very different than the requirements used for non aerospace applications. A primary reason is because of the environments in which the materials and processes are used. Aerospace environments can include atomic oxygen and radiation environments of low earth orbit, severe vacuum, higher temperatures, and the radiation environments beyond low earth orbit and other environments such as the corrosive environments of our seacoasts, embrittlement from gaseous hydrogen and liquid metals, complex aeroheating and flow environments, high engine temperatures, moisture, fungus, and cryogenic liquids. The materials used to fabricate flight hardware should be selected only after considering the operational requirements for those unique applications, and also selected after considering the design engineering properties of the candidate materials. In addition, we are faced with unique hardware that has usually been designed to lower factors of safety-not because we lessen hardware safety and reliability, but because of the stringent performance requirements as needed by the design. Aerospace factors of safety are normally 2.0 or below while most construction or commercial hardware are designed using factors of safety of 10 or more-structural efficiency is a major design driver. Another major driver is safety and reliability. If we fail in aerospace, people die and we cost the taxpayers billions of dollars. Simply put, we cannot fail. Performance, safety and reliability, and the complexity of our missions drives the designs towards specialization-again, the hardware many times is unique and one of a kind. And every step that is used in the production of every material and process we use must be verified and documented. And as an end result of all of this, aerospace hardware can be and usually is extremely expensive.

"How can we reduce the cost of the hardware?" is a question many have asked time and time again. While we do not have all the answers, we do have methods that can minimize costs and at least move towards cost efficiency. It is proposed by this author that three ways to help bring cost efficiency into the picture for aerospace hardware include requirements, applying Design for Manufacturing techniques during hardware design, and conducting the necessary development before starting production. 


\section{Requirements Discussion}

A good requirement has four primary characteristics: 1) it must be clear, concise, unambiguous, and complete 2) it must be measurable and quantifiable 3) it must be achievable and last but not least 4) it must be necessary! of course, if the requirement is not necessary, it should not be imposed. The first three are also somewhat interdependent. It is fundamental and extremely important for requirements to be easily understood and unambiguous - you have to convey your requirement in a language and structure that your audience can understand it. What good is a requirement that no one can understand? It is also important to understand your audience. Is it intended for the requirement to be understood by a materials engineer, design engineer, or an engineering technician? Each of these three entities speaks and interprets technical language differently. The primary reason that it is important that a requirement be measurable and quantifiable is that all requirements must be verified. How can you verify that a requirement has been met if you cannot quantify or measure it? If you cannot verify your requirement it will be almost impossible to determine whether the requirement has been met. When this dilemma surfaces in aerospace, we use waivers and rationale to justify not meeting the requirement. This process can be time consuming, expensive, and may not always produce a desired result. Now, let us think about the third characteristic-what good is a requirement if it cannot be achieved? This is where we at NASA have failed time and time again, spouting out requirements that cannot be achieved or extremely difficult to achieve. It is closely related to the second characteristic. The common problem is that there is a train of thought that believes that requirements are goals. Requirements are not goals-goals are something that you hope to achieve, requirements are tangible and something you had better achieve or in our business, you will fail.

Now how do you know whether the requirement can or cannot be achieved? How do you make sure that your requirement has met these four characteristics? This is where I believe our laboratory's approach to materials and processes requirements is, though not necessarily unique, but definitely a step in the right direction. You know that many requirements can be achieved from experience and common practice. Let us explore a relatively simple example, we can write a requirement for a material certification as: "The vendor shall deliver a Certificate of Compliance for each part which shall, as a minimum, include the alloy chemistry, tensile and fracture toughness properties of witness specimens from the same material heat and lot". (Please remember that this is purely an example, not intended to be a complete aerospace requirement.) Is this requirement clear, concise, unambiguous, and complete? To me, as a metallurgist, it is clear. I know what a chemical analysis is; I know how to conduct a tensile test and a fracture toughness test. I am not telling the vendor how to do these tests (though I could call out the specifications), but I'm telling them that they have to deliver a Certification of Compliance (CofC) and of what that CofC has to consist. I do need to consider, however, who my audience is and if the requirement is working within the frame of reference for all material procurements. Chemical analysis, tensile and fracture properties, witness specimens, etc. have been part of material certifications for many years. The expectation I have for my audience is that they are cognizant of how industry normally conducts business. If I had specified something out of the ordinary, it is up to the author of the requirement to clearly and succinctly convey this new requirement to his audience. Is this requirement measurable and quantifiable? Chemistry, tensile and fracture toughness are all standard quality characteristics for alloys- the values that will be presented in the CofC will demonstrate if the part is within the specified chemistry, tensile and fracture properties. Is it achievable? Yes, if the recipient of the requirement plans ahead, but it is extremely important that the author of the requirement work with the recipient before the requirement is levied. In this particular case, the use of witness coupons from the same lot as the part is being specified. A part is not being sacrificed to be cut up and tested, rather the vendor plan ahead to allow some of the material from the part lots to be used for witness coupons. If the vendor does not plan ahead, a part will have to be sacrificed or a waiver will have to written. I also know from experience that a chemical analysis, tensile properties, and fracture toughness properties can all be obtained from witness coupons that come from the same lot and/or heat of material if we plan ahead.

Now, is the requirement necessary? Here's where some engineering judgment comes in where we must do our homework. Many times we will levy a requirement for a material that will include chemistry, tensile properties, and fracture toughness properties without thinking about the part's end use or how the part will be utilized in the design. This is where we as materials engineers must do their job. I need to know the chemistry is within specification limits so that I will know that the material is indeed the material that I specified. There are alloys that have different chemistries but have similar tensile and fracture properties. I need to know the room tensile properties because I have to guarantee that the design properties can meet the required factors of safety. I need to know the fracture properties (in this case) so that the design can predict the life of the part and its tolerance to damage. You will note that room temperature tensile and fracture properties were specified. This is also where materials engineers must their homework. If the part is going to be used in a cryogenic environment or a corrosive environment, I'd better have done my homework in knowing that if my material meets these room temperature requirements that we have 
historical property characterization or knowledge that demonstrates that if the material performs at this quality level at room temperature, it will perform at a certain quality level in that cryogenic environment or that corrosive environment. Yes, the cryogenic properties and corrosion properties can be specified as a deliverable for the CofC for that part, but is it really necessary? Have I selected a material that is that unpredictable? Have I selected a material for an environment where I don't know how it will perform? Ultimately, it is important for the materials engineer to do his homework, select the right material for the right environment and do the proper amount of "homework" such that we know that the material will perform as needed by the design.

The method that our laboratory has used successfully is to specify as many and as complete a set of detailed requirements up front before or at least during the initial stages of design. This includes bringing all the "lessons learned" or guidelines documents for consideration only (not to be implemented) along with requirements that may or may not be applicable to the design. Now this sounds somewhat contrary to what was discussed earlier, in fact it could be interpreted as specifying "unnecessary" requirements up front. However, the important difference is that you have to work your requirements ahead of time with the recipient of the requirement to make sure the requirements meet the four characteristics. We do not just "throw the requirement over the fence" without receiving feedback. The method that our laboratory uses is called requirements tailoring.

Requirements tailoring is a process where the requirements organization and the vendor work together to mutually determine what requirements are applicable and which are not, how the requirements will be met, how the requirements will be implemented, and how the requirements will be verified and controlled. In doing so, requirements may be tailored for specific programs or projects simply by constructing a matrix of applicable paragraphs and paragraphs that are not applicable, subject the approval of the of the responsible NASA Materials \& Processes organization. Tailoring also includes mutually agreeing to use existing or previously developed contractor processes and standards in lieu of government specified processes and standards. The philosophy here is that many vendors or contractors already have established processes, and in the great majority of those cases, these processes will meet or exceed the imposed requirements. The tailoring of requirements may be documented in a Materials and Processes Selection, Implementation, and Control Plan, by providing the degree of conformance and the method of implementation for each of the identified requirements. If done properly, requirements tailoring can result in mutually understood, mutually negotiated and mutually agreed to set of requirements. When conducted properly up front in a program, costs, schedule and technical are much more easily defined and understood. Issues or problems can be identified before hardware is built-specifying requirements up front allows us to initially consider the known-unknowns and possibly allow us to start thinking about the unknown-unknowns which will be discussed in the last section. Using requirements tailoring can result in cost efficiency and higher quality hardware.

\section{Design for Manufacturing Discussion}

Design for Manufacturing (DFM) principles should be used when designing and developing hardware. Yes, it is easy to make this statement but difficult to do with aerospace hardware. Regardless, we must be cognizant of these principles during the design process in order to achieve cost efficiency. Implementing these principles will be a dilemma faced as we design our new Crew Launch Vehicle. Design for Manufacture (DFM) is a set of techniques or a method used for efficient production, assembly, and testing of a product through the manufacturing process. The DFM objective is to produce a design that implies producability or ease of manufacturing at a reasonable cost while satisfying the functional requirements of the part, component or assembly. ${ }^{\dagger}$ We will now review a few of the highlights of DFM principles that can be applied to aerospace hardware.

Simplification is a DFM technique that can be summarized in three words: eliminate, simplify, and standardize. An optimal design should minimize product's manufacturing information within the constraints of that part's function and performance, therefore we should always attempt to eliminate unnecessary parts or consolidate/combine parts. When we do this, there is a ripple effect on cost and quality-fewer unnecessary parts mean that there are fewer parts to manufacture, less material handling, and therefore fewer parts and components that must be planned for and controlled through the manufacturing process. A major part of simplification involves minimizing part variations. Part standardization for shape and dimensions simplifies assemblies and reduces manufacturing costs because of reduced tooling and setup, and longer production runs. The cost of incorporating a fastener in the design can be more than the fastener itself, so only use fasteners when absolutely necessary. Maximize the overall ease of subassembly by designing to a base subassembly, and also minimize and eliminate

${ }^{\dagger}$ Bralla, James G. Design for Manufacturability Handbook, 2nd Edition. New York: McGraw Hill, 1999. 
unnecessary subassemblies. Bottom line is to consider how the parts will be assembled during design by using chamfers, slots, and/or tapers. It is also advisable to use the talents of a skilled manufacturing engineer during the design.

Parts or components should be standardized whenever possible by minimizing the use of special tools and fixtures, designing to the axis of insertion along the line of sight, using self locating features, and reducing adjustments by considering tolerances carefully. Keep in mind that standardization includes materials, machinery, and the design. Make every attempt to use commonly available, standard materials and components, and available machinery. Even though aerospace hardware can be extremely complex, parts can still be standardized and simplified.

Materials selection and processes selection should not be considered independently and the selections should be considered integral to the design process. Materials selection should be organized into at least three categoriesproperties, specifications, and data for ordering. It should be noted that the number of manufacturing processes required to complete the part can be the greatest single determinant of direct labor cost. In addition, consider the use of lower labor processes such as metal stamping and drawing, die casting, injection molding, single-spindle and multi-spindle machining, and processing and packaging in which secondary work can be limited to one or two operations. In contrast, make every attempt to minimize use of higher direct labor processes such as conventional machining, investment casting, mechanical assembly including adjustment and calibration.

Process restrictiveness can be avoided by specifying the required design characteristics, not the process. Taking advantage of special process characteristics can eliminate unneeded operations and the need for separate components. Materials that are easily processed should be used whenever possible which includes materials that are easily machined, don't require special handling, have longer shelf life, are readily available, and not difficult to repair.

It is also advisable to design for low labor cost processes and the first step should be to consider the function of the design characteristic. Using processes of a known capability minimize costly development, special instructions, additional quality control and verification. Some rules of thumb include using processes within the realm of manufacturing, using new technology only when necessary and making sure the cost and schedules required for any new technology are considered in the design decisions. This also includes making sure that the development time required for maturing and implementing the technology is considered.

Using parts at de-rated values with no overstress is a DFM technique for the handling of parts during the manufacturing processes. In order to accomplish this, the processing, handling, assembly, and residual stresses for each individual part should be considered. Minimize the handling of parts and minimize stresses by increasing thickness or tooling. The over handling of parts may increase cost and lower quality. Human factors should be considered. A good rule of thumb is that the maximum weight a person should handle is about 50 pounds. Bulky, rangy, and flexible components can be difficult to handle and should be considered in the initial part design rather than on the manufacturing floor or even while developing the assembly drawings.

The type and number of operations needed to produce the product and quantities should be minimized. All factors being equal, the product with the fewest number of operations required for manufacture will usually be the most efficient to manufacture, therefore less costly and more simple to manufacture. Operations of known capability are also easier to produce. It is important to design a product so that as many operations as possible can be performed without repositioning which minimizes handling and set up time. Secondary operations should be avoided and/or streamlined. Operations such as deburring, inspection, and heat treating add cost and time.

Tolerances can be defined as the total amount by which a given dimension may vary, or the difference between the limits and represent the functional requirements that need to be maintained for a product to function properly. Uniform dimensions can be a key factor in reducing unit cost. The goal should be to provide the necessary control of selected locations to ensure a quality assembly and to meet specified requirements. The general rule is to only specify tolerances necessary for the part to function as needed. Whenever possible use liberal tolerances, uniform dimensions, consider part interchangeability, use manufacturing processes that can meet the desired tolerances, and dimension from specified surfaces only which can help avoid tooling, gage, measurement, and inspection errors.

In summary, many of the DFM techniques as stated above are indeed difficult to implement for the manufacture of aerospace hardware. But again, many times we as engineers do not take a simplistic approach during design-it is just not trendy. We must overcome our need to think that all manufacturing processes have to be high tech just because it is aerospace hardware. 


\section{The Marriage of Design and Materials Discussion}

Design is the cornerstone for how our aerospace hardware will perform. I do know from experience that materials are fundamental to the design, and materials have to be considered a major component of design. We have a saying in the materials lab: "It's awfully difficult to build aerospace hardware without materials". It does seem to be a statement that maybe overemphasizes materials, but it is true in the aspect that design and materials are fundamental to building aerospace hardware, and they are not mutually exclusive, they are codependent. In addition, we have to make sure that we do the proper amount of development to make sure that our design and materials that the design will use can be verified and validated. Verification tells us that the design will perform as designed or required. Validation tells us that the design is indeed the correct design and it will meet the mission requirements.

A material cannot be properly selected for a particular design without knowing the environments that the hardware is expected to perform. The life requirements must also be known. Sound engineering judgment is required to determine how verification will be accomplished. Will verification be by test, analysis, or similarity? In aerospace, a large percentage of the hardware is unique. Unique hardware cries for verification by test and should be the dominate selection between these three choices. Part, component, element, subsystem, or system testing verifies the analysis. Analysis creates a model that allows us to predict how the design will perform. This involves proper material selection and enough material development testing to generate the properties needed for the design and analysis of the hardware. Design and analysis cannot take place without characterized materials. You just can't design and analyze without material properties. Because of the uniqueness of aerospace hardware, it is difficult to verify that hardware without testing.

Traditionally, design is about $5 \%$ of the total cost, but design efforts influence $70 \%$ of the manufacturing cost. Hasty study periods, resulting in flawed or incomplete requirements, are a major cause of project failure. The GAO reported in 1999 that high tech government projects continue to fail for low-tech reasons. Typical low tech reasons are flaws built in through incomplete study periods and improper implementation of an otherwise sound project management process. ${ }^{\ddagger}$

Aerospace hardware is expensive for a myriad of reasons, but it should be expensive only because of its uniqueness and that the hardware "pushes the limits" of technology and mission. It should not be expensive because we do not do our homework. Many times in aerospace we are constrained by cost and schedule and at times can be our own worst enemy. A material or process is selected because it is "trendy" or "sexy" to do so, or we overestimate its technology readiness level. A final decision on material selection should never be made until the mission and design requirements are known - that is, the environmental and life requirements. And we should always, always, always assure that the material is properly characterized for those environmental and life requirements.

Failure can be described in two ways. There is the conventional hardware failure that results in loss of function, mission, or life. But there is a second type of failure that has to be considered when dealing with hardware and that is failure of the hardware to operate efficiently. If your hardware performs as designed and meets the mission requirements but is too costly to operate, this has to also be considered failure. Design influences $70 \%$ of the manufacturing costs and design is only $5 \%$ of the total cost. Look at this leverage-if we design without proper material and manufacturing considerations we have failed. Failure can mean that the hardware will be too expensive to manufacture, maintain, and operate.

The other aspect of failure is the underlying safety system concerns that may exist. These underlying concerns are what I will define as the unknown unknowns. Development can be approached with three design aspects: the known knowns, the known unknowns, and the unknown unknowns. All three aspects of design have to be considered and planned for in a project. A known known is where we know what the requirement is and we know how to meet that requirement. Known knowns can be addressed by analysis and/or test. However, we HAVE to address the known unknowns by test. A known unknown is where we know what the requirement is, but we are not certain how to achieve it--in other words, some type of development is necessary. The interesting aspect of this is that if these known unknowns are not addressed by test, it will be very difficult to gaining enough insight to start addressing the unknown unknowns. An example of this was the Space Shuttle Solid Rocket Booster Aft Skirt failure. The original aft skirts were tested to $140 \%$ of their design loads in the 1970's during the Space Shuttle development. In the mid-1980's MSFC was testing a new development Reusable Solid Rocket Motor case segment (a filament wound case segment as a replacement for the existing D6AC alloy steel case segments) using an excessed aft skirt as a test fixture. This aft skirt failed at $128 \%$ of design loads. A subsequent aft skirt was tested and failed around $130 \%$. I did not experience what the design considerations were for the aft skirt during the original

\footnotetext{
${ }^{\ddagger}$ Forsberg, Mooz, and Cotterman. Visualizing Project Management. New York: John Wiley \& Sons, Inc., 2000
} 
development, but did play a key part of the failure investigation and subsequent "fix". Our laboratory focused on two areas of development for the original design - the hold down post forging development and characterization and the weld development which included the development of the weld properties. The hold down post was very well characterized-development focused on the manufacture of some of the largest 2219-T352 aluminum hand forgings to date. The development focus centered on attaining acceptable cold work in the thickest areas (which were at the aft end of the aft skirt) to achieve the highest tensile strengths possible. The weld development centered on characterizing weld strengths of both fore and aft sections of the hold down post forgings. As an end result, the final design configuration had the lowest strength forging areas and the lowest weld strengths are in the area of this thicker section of the hold down post forging. The problem with this is that these are areas of the aft skirt which undergo the highest stresses. Compounding this situation is that because we were pushing the envelope on forging size, the weld land in this high stress area was the shortest length of the longitudinal welds along the aft skirt. This short weld land created a stress concentration area. When all of this is taken in consideration, the aft skirt hold down post forging welds were underdesigned, and even though the original structural test article "passed" the qualification load limit, there was only one test article tested until the filament wound case test. In hindsight, additional testing should have been required. The parent material and weld properties were well characterized and the design "on paper" was properly designed, but it was the unknown-unknowns which was not given enough consideration. (Note: final redesign was to add a doubler across the high stress weld area).

\section{Conclusion}

In summary, this paper has outlined some of the more important aspects of using materials and processes requirements in the design process. The central theme of the information presented here is that we, as engineers, have to spend more time up front in programs defining requirements, considering manufacturing processes during the design process, developing necessary technologies, and conducting the proper and needed amount of testing to verify our aerospace hardware. There were four statements footnoted earlier which provide a historical justification as to the criticality of the study phase: "Traditionally, design is about $5 \%$ of the total cost, but design efforts influence $70 \%$ of the manufacturing cost. Hasty study periods, resulting in flawed or incomplete requirements, are a major cause of project failure. The GAO reported in 1999 that high tech government projects continue to fail for low-tech reasons. Typical low tech reasons are flaws built in through incomplete study periods and improper implementation of an otherwise sound project management process." In other words, our philosophy should be to do it right the first time, or at least make every attempt to do it right the first time.

\section{References}

${ }^{1}$ Bedworth, Henderson, and Wolfe. Computer Integrated Design and Manufacturing.

${ }^{2}$ Boothroyd, Poli, and Murch. Automatic Assembly. New York: Marcel Dekker, 1982.

${ }^{3}$ Bralla, James G. Design for Excellence. Washington D.C.: McGraw- Hill, Inc., 1996.

${ }^{4}$ Bralla, James G. Design for Manufacturability Handbook, 2nd Edition. New York: McGraw Hill, 1999.

${ }^{5}$ Clements, Richard Barret. Handbook of Statistical Methods in Manufacturing. Englewood Cliffs, New Jersey: Prentice Hall, 1991.

${ }^{6}$ Crevling, C. M. Tolerance Design. Redaing, Massachusetts: Addison - Wesley, 1997.

${ }^{7}$ DeGarmo, Black, and Kohser. Materials and Processes in Manufacturing, 6th Edition. New York: MacMillan

Publishing Company, 1984

${ }^{8}$ Ettlie and Stoll. Managing the Design-Manufacturing Process. New York: McGraw-Hill, Inc., 1990.

${ }^{9}$ Forsberg, Mooz, and Cotterman. Visualizing Project Management. New York: John Wiley \& Sons, Inc., 2000

${ }^{10}$ Himmelfarb, Philip A. Product Development During the 90's. Englewood Cliffs, New Jersey: Prentice Hall, 1992.

${ }^{11}$ Kassapoglou, Christos. "Determination of the Optimum Implementation Plan for Manufacturing Technologies-

The Case of a Helicopter Fuselage." Joumal of Manufacturing Systems Volume 19No. 2, 2000. p 121-133

${ }^{12}$ Leaney, P.G. \& G. "Design for Assembling: The Evaluation Methods of Hitachi, Boothroyd, and Lucas."

International Journal of Assembly Automation. Cleveland: Wittenberg, 1992.

${ }^{13}$ Liggett, John V. Fundamentals of Position Tolerance. Dearborn, Michigan: Society of Manufacturing Engineers, 1970.

${ }^{14}$ Mabee, David G., Bommer, Michael, and Keat, William D. "Design Charts for Remanufacturing Assessment." 
Journal of Manufacturing Systems. Volume 18/ No. 5, 1999. p 358-366

${ }^{15}$ Miller, Stanley S. Competitive Manufacturing-Using Production as a Management Tool. New York: Van Nostrand Reinhold Company, 1988.

${ }^{16}$ Pedley, Michael, Vaughn, Timothy, JSC 49774 Standard Manned Spacecraft Requirements for Materials and Processes, July 2003

${ }^{17}$ Salvendy, Gavriel. Handbook of Industrial Engineering. New York: Wiley, 1982.

${ }^{18}$ Sule, D.R. Manufacturing Facilities, Location, Planning and Design. Boston: PWS Publishing Company, 1994.

${ }^{19}$ Timpe, A. Dale. Productivity, the Art and Science of Business Management. New York: Kend Publishing Company, 1989.

${ }^{20}$ Vaughn, Timothy, Materials and Processes Design Guide Handbook For Space Systems, December 2005

${ }^{21}$ Walker, Jack. Handbook of Manufacturing Engineering. New York: Marcels Dekker, 1996. 


\title{
Manned Spacecraft Requirements for Materials and Processes
}

\begin{abstract}
A major cause of project failure can be attributed to an emphasized focus on end products and inadequate attention to resolving development risks during the initial phases of a project. The initial phases of a project, which we will call the "study period", are critical to determining project scope and costs, and can make or break most projects. If the requirements are not defined adequately, how can the scope be adequately determined, also how can the costs of the entire project be effectively estimated, and how can the risk of project success be accurately assessed?

Using the proper material specifications and standards and incorporating these specifications and standards in the design process should be considered inherently crucial to the technical success of a project as just as importantly, crucial to the cost and schedule success. This paper will intertwine several important aspects or considerations for project success: 1) Characteristics of a "Good Material Requirement" 2) Linking material requirements to the implementation of "Design for Manufacturing" techniques and 3) The importance of decomposing materials requirements during the study phase/development phase to mitigate project risk for the maturation of technologies before the building of hardware.
\end{abstract}

